\title{
Spontaneous cervical artery dissection in patients aged over 70 years: two cases and systematic literature review
}

This article was published in the following Dove Press journal:

Clinical Interventions in Aging

26 August 2017

Number of times this article has been viewed

\author{
Nolwenn Riou-Comte' \\ Gioia Mione' \\ Lisa Humbertjean' \\ Marie-Alexia Ottenin ${ }^{2}$ \\ Jean-Christophe Lacour ${ }^{\prime}$ \\ Sébastien Richard ${ }^{1,3}$ \\ 'Department of Neurology, Stroke \\ Unit, University Hospital of Nancy, \\ Nancy Cedex, France; ${ }^{2}$ Department \\ of Neuroradiology, University \\ Hospital of Nancy, Nancy Cedex, \\ France; ${ }^{3}$ Centre d'Invesigation \\ Clinique Plurithématique Pierre \\ Drouin CIC-P I433 INSERM UIII6, \\ University Hospital of Nancy, \\ Vandoeuvre-lès-Nancy, France
}

Correspondence: Sébastien Richard Unité Neurovasculaire, Service de Neurologie, Hôpital Central, 29 avenue Maréchal de Lattre de Tassigny, 54000 Nancy, France

Tel +33 383852256

Fax +33 38385 II 59

Email s.richard@chru-nancy.fr
Objective: Spontaneous cervical artery dissection (CAD) is a cerebrovascular disease typically considered to affect the young population. Literature reports cases in the elderly only as incidental findings, making the diagnosis unlikely in older patients. Incidence and pathogeny in this specific population remain to be assessed.

Methods: We reviewed patients aged over 70 years admitted for spontaneous CAD in the Stroke Unit of the University Hospital of Nancy (northeastern France) over a period of 12 years as well as all reported cases in literature.

Results: During this period, only two patients aged over 70 years were diagnosed with internal carotid artery dissection in our center. The first patient was diagnosed with the typical radiological feature of long tapered stenosis due to mural hematoma. The second patient presented with the classic painful Horner syndrome. Literature review identified only two case reports and eight studies with an age range above 70 years. Headache was present in nearly all documented cases. Radiological features were the same as those usually described in younger patients.

Conclusions: Even if spontaneous CAD in patients aged over 70 years would appear to be rare, it does occur with comparable clinical and radiological features as in the younger population. $\mathrm{CAD}$ is probably underdiagnosed in this population due to a higher prevalence of more common causes of stroke at this age. However, a simple investigation into headache or the Horner syndrome during the patient's diagnostic workup would lead to adapted exploration of cervical arteries and improve detection of CAD in the elderly.

Keywords: cerebral infarction, elderly, false aneurysm, Horner syndrome, old patients, spontaneous cervical artery dissection

\section{Plain language summary}

Spontaneous cervical artery dissection (CAD) is defined as a tear within the vessel wall and the consequent formation of hematoma. While it is a well-known common etiology of ischemic stroke in younger patients, it remains poorly reported in the elderly. As a result, most physicians do not consider CAD during the diagnostic workup in elderly patients presenting with cerebral infarction. We reviewed all the cases of CAD in patients older than 70 years admitted to our stroke unit over a period of 10 years and studied all reported cases in literature. We identified two patients in our center during the study period, and 10 papers in the literature describing patients older than 70 years who presented with CAD. Clinical presentation involved headache in nearly all of the documented reports and the classic ophthalmic Horner syndrome in one case. The radiological features were the same as for younger patients with the characteristic arterial stenosis and dilation due to hematoma. In conclusion, physicians should be aware that spontaneous CAD is a possible 
cause of stroke in the elderly patient and investigate for headache and Horner syndrome. This is all the more important as patients with $\mathrm{CAD}$ require specific management and monitoring.

\section{Introduction}

CAD is defined as the formation of hematoma within the vessel wall possibly involving embolism, occlusion of the vascular lumen, and external compression depending on its location. ${ }^{1}$ It is now a well-known common etiology of ischemic stroke in younger patients and is systematically part of the diagnostic workup in this population. ${ }^{1}$ In contrast, physicians rarely explore elderly stroke patients for CAD due to the higher frequency of other obvious causes of stroke such as atherosclerosis and atrial fibrillation. Reported cases are rare but demonstrate that $\mathrm{CAD}$ can be a cause of stroke in the elderly. ${ }^{2,3}$ A registry of clinical and radiological data of proven cases would be of interest to determine specific features of $\mathrm{CAD}$ in this population. It would help to diagnose this important etiology of stroke, which requires adapted management, and to measure the real incidence. Here, we describe two cases of patients older than 70 years with CAD admitted in our Stroke Unit over a period of 12 years and review reported cases in literature.

\section{Methods}

We conducted an observational, retrospective study of patients older than 70 years diagnosed with spontaneous CAD admitted to the Stroke Unit of the University Hospital of Nancy (northeastern France) from January 1, 2004 to December 31, 2015. Patients were identified from our institutional anonymized database.

A search of reported cases in the literature was performed using the database Medline/Pubmed for articles published over the last 30 years with the keywords: "cervical artery dissection" and "spontaneous cervical artery dissection". We extracted case reports of patients older than 70 years and studies with an age range over 70 years. Cases describing intracranial dissections only, atherosclerotic aneurysm, and dissection due to proven traumatism, surgery, or cancer were excluded. We report data about the type of paper, demographic information, medical history, symptoms, radiological features, treatments, and outcome.

The study was observational without any intervention on human beings or animals. All data were anonymized. Approval was obtained from the ethics committee of the University Hospital of Nancy (France). Both the patients from our center gave written consent for the publication of their medical history and images.

\section{Results}

During the study period, two patients older than 70 years with spontaneous CAD were admitted to our Stroke Unit. This represents $<0.1 \%$ of overall patients seen in the Stroke Unit and $<0.1 \%$ of patients over 70 years diagnosed with CAD. Literature review found only eight studies about CAD including a population with an age range over 70 years, ${ }^{4-11}$ and two case reports describing a 70- and an 80-year-old patient with spontaneous CAD. ${ }^{2,3}$ Data from these references and our cases are detailed in Table 1. Out of all the reported cases, the sex was known for five patients of whom two were men and three were women. The oldest patient was 89 years. Location of CAD was documented in 10 cases - the internal carotid artery (ICA) for six patients, the vertebral artery for three patients, and dissection of the common carotid artery (CCA) for one patient. The patients' medical histories were detailed only in the two case reports in addition to ours. One of these patients presented no vascular risk factors other than age and two previous symptomatic cerebral infarctions. ${ }^{2,3}$ Clinical presentation is described in six cases and involved headache in five. One patient presented with painful Horner syndrome due to ICA dissection. All other signs were caused by cerebral infarction. Radiological features are described in five cases. Arterial stenosis or occlusion were observed in four cases and false aneurysm in two (one patient presenting both with the pearl-and-string sign). Treatment and outcome are poorly reported with only five documented cases. Antithrombotic therapy was used in three cases (two with heparin and one with antiplatelet therapy), and interventional neuroradiology was required in one patient presenting a giant dissecting aneurysm of the ICA. Outcome seems to be good with only one death following posterior circulation stroke and no documented cases of recurrence of cerebral infarction or CAD.

\section{Case I}

A 77-year-old man with a medical history of arterial hypertension and dyslipidemia was admitted because of a 90-minute episode of aphasia accompanied by sudden headache. Neurological examination at admission was normal except for continuation of cephalalgia. Blood tests showed hemoglobin level of $16 \mathrm{~g} / \mathrm{dL}$, platelet count of $220 \mathrm{G} / \mathrm{L}$, $\mathrm{C}$-reactive protein level under $5 \mathrm{mg} / \mathrm{L}$, low-density lipoprotein cholesterol level of $3.2 \mathrm{mmol} / \mathrm{L}$, preprandial blood glucose level of $4.5 \mathrm{mmol} / \mathrm{L}$, and normal coagulation tests. Electrocardiogram and 48-hour monitoring failed to reveal arrhythmia. Brain computed tomography (CT) scan and lumbar puncture were first performed to rule out subarachnoid 


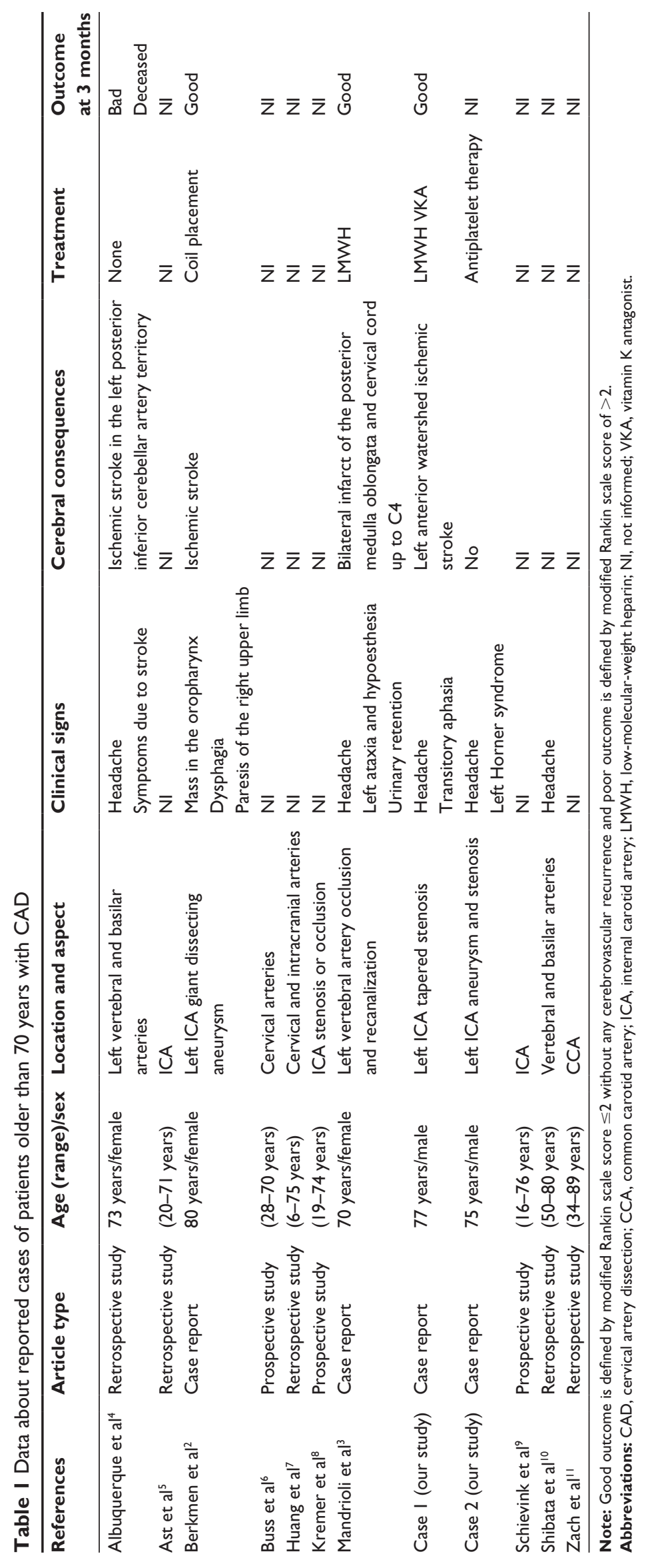


hemorrhage. No signs of hemorrhagic or ischemic lesions were observed; cerebrospinal fluid was clear with normal values. Exploration of the cervical and intracranial arteries by ultrasound did not show any atherosclerotic lesions but revealed high resistive index values in the left CCA and ICA with decreased cerebral blood flow in the supply arteries (Figure 1). Brain CT angiography confirmed an extremely tight stenosis remote from the left ICA bulb and extended over $2 \mathrm{~cm}$ (Figure 2A), with a 30\% increase in artery diameter compared with the right ICA (Figure 2B). Brain magnetic resonance imaging (MRI) showed high-intensity signal on cervical axial fat-suppressed T1-weighted sequence proving the presence of hematoma at the level of stenosis (Figure 2C) and recent left anterior watershed infarction on diffusionweighted sequence (Figure 2D). Infarction was also seen on fluid-attenuated inversion recovery (FLAIR) sequence and former lesions due to hypertensive vasculopathy. In view of this presentation compatible with CAD, anticoagulation therapy was started with low-molecular-weight heparin and rapid overlap with vitamin $\mathrm{K}$ antagonist. A control brain $\mathrm{CT}$ angiography performed 3 months later showed an occlusion of the ICA preceded by a long tapered stenosis of typical CAD aspect. Anticoagulation therapy was replaced with a daily dose of aspirin without any cerebral ischemic recurrence during 2 years of follow-up.

\section{Case 2}

A 75-year-old man with a medical history of ankylosing spondylitis, arterial hypertension, and several ischemic strokes consulted his regular neurologist because of recent headaches. The patient was taking nonsteroidal anti-inflammatory drugs but had discontinued clopidogrel several weeks previously. Medical interview did not reveal any recent infection. Neurological examination showed a left Horner syndrome with no neurological deficit (Figure 3). A brain MRI with angiography of the cerebral arteries was performed which
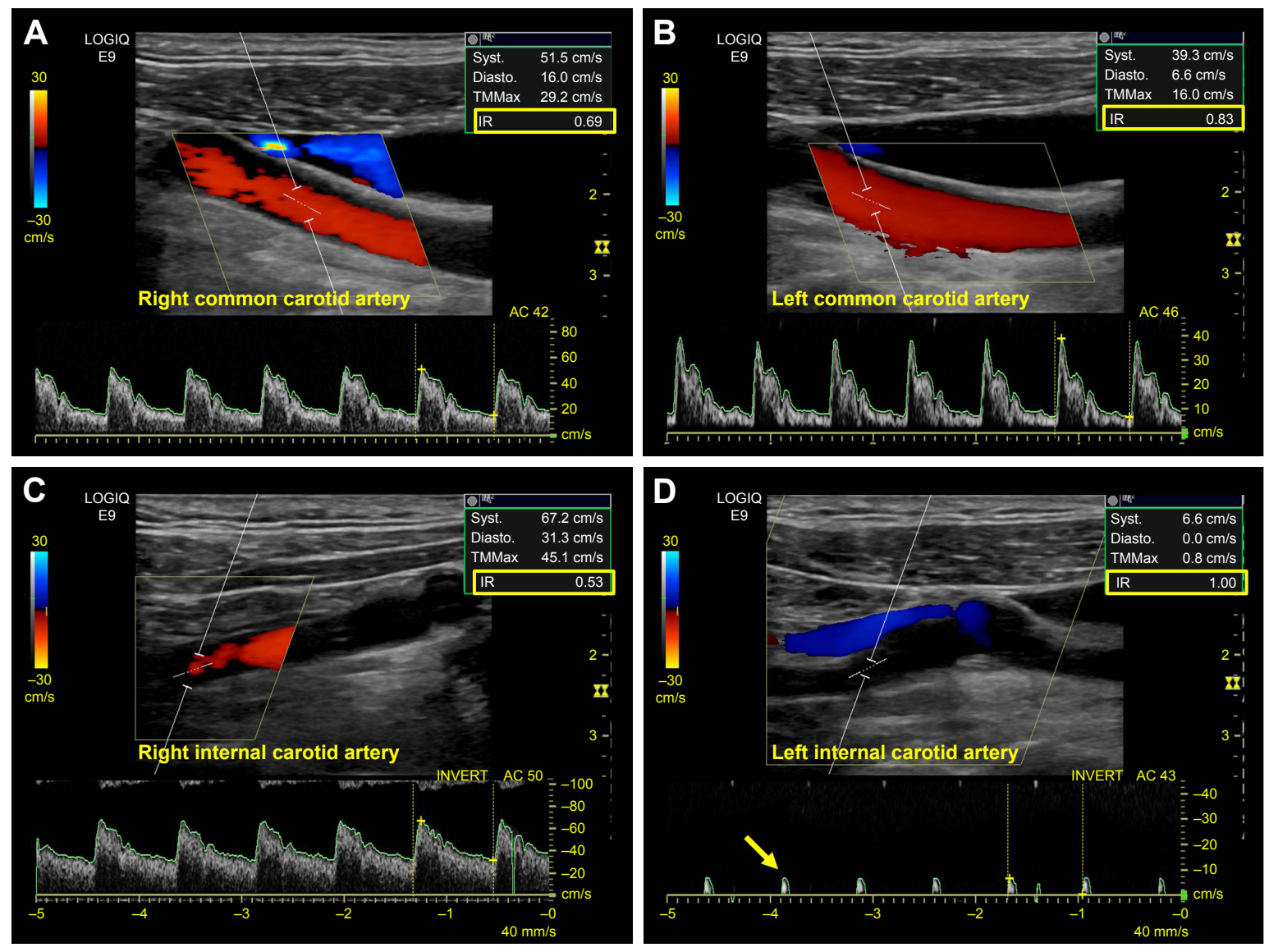

Figure I Ultrasonography investigation with diagnosis of left ICA dissection (case I).

Notes: (A) Right CCA; (B) left CCA; (C) right ICA; (D) left ICA; resistive index values are underlined - increased in the left CCA (0.83) compared with the right CCA (0.69) and in the left ICA (I.00) compared with the right ICA (0.53); absence of blood flow in the left ICA (arrow).

Abbreviations: CCA, common carotid artery; ICA, internal carotid artery. 

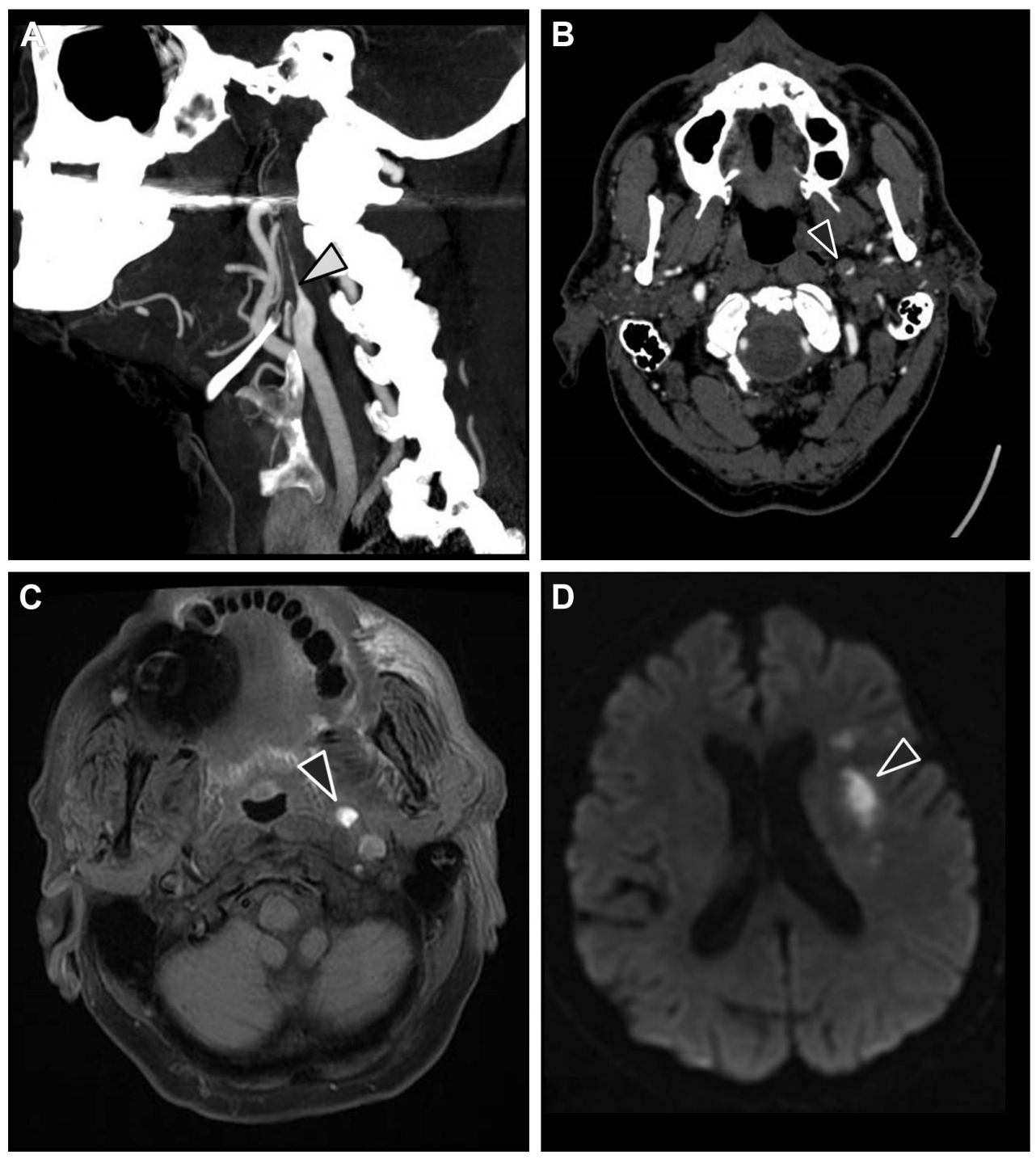

Figure 2 Cervical CT angiography and brain MRI with diagnosis of left ICA dissection and cerebral infarction (case I).

Notes: (A) Cervical CT angiography showing long tapered stenosis (arrow) of the left ICA; (B) axial cervical CT angiography showing stenosis with narrowing of left ICA lumen and increased arterial diameter by hematoma (arrow); (C) axial fat-suppressed TI-weighted MRI showing hematoma in the left ICA wall (arrow); (D) diffusionweighted MRI showing recent left infarction (arrow).

Abbreviations: $\mathrm{CT}$, computed tomography; ICA, internal carotid artery; MRI, magnetic resonance imaging.

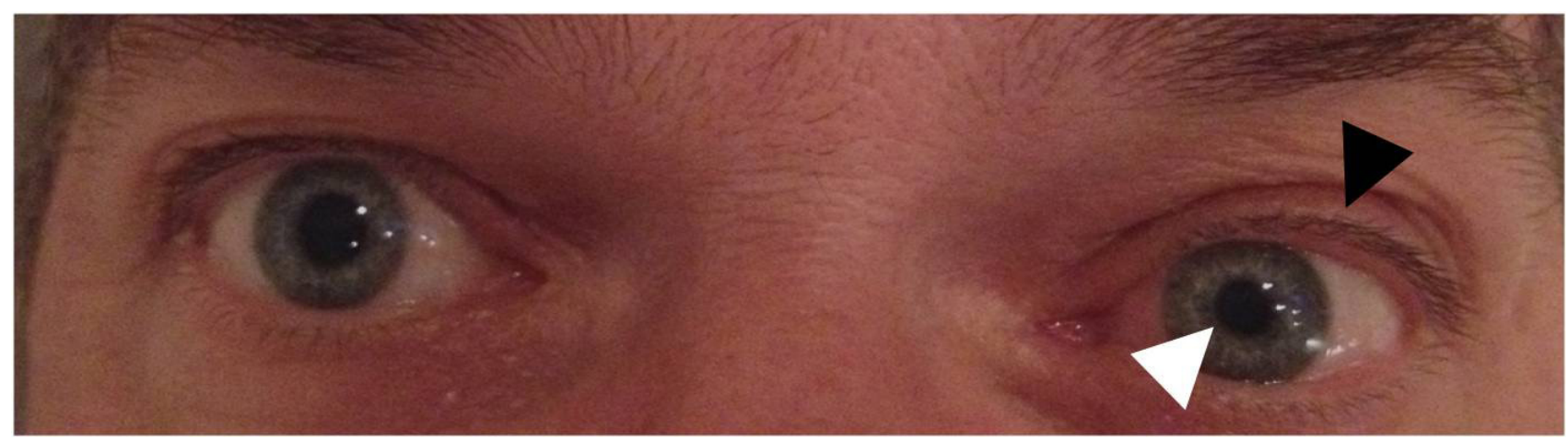

Figure 3 Left Horner syndrome.

Note: Miosis (white arrow) and ptosis (black arrow). 

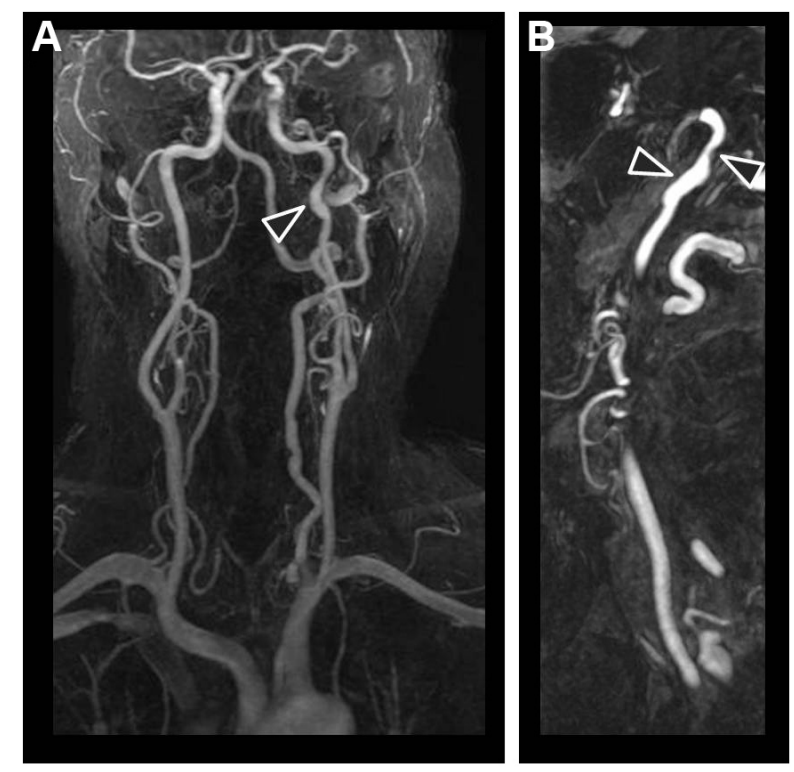

Figure 4 Cervical MR angiography with diagnosis of left ICA dissection (case 2). Note: (A, B) Cervical MR angiography showing aneurysmal dilation topped with stenosis (pearl-and-string sign) (arrows).

Abbreviations: ICA, internal carotid artery; MR, magnetic resonance.

revealed an aneurysmal dilation topped with a stenosis in the subpetrous portion of the left ICA (Figure 4A and B) without any cerebral ischemic consequences on diffusion-weighted sequence or former infarctions on FLAIR sequence. The usual laboratory tests had not been performed. Exploration for atherosclerotic lesions found only a mild plaque at the origin of the right vertebral artery. Management consisted of resumption of antiplatelet therapy with ultrasonographic control of cervical and intracranial arteries. Left Horner syndrome and headache resolved after several weeks and the patient did not experience any cerebrovascular events during a follow-up of 7 years. The patient was unwilling to undergo control CT or MR angiography because of severe chronic back pain due to ankylosing spondylitis.

\section{Discussion}

The incidence of CAD in the general population is very low (about 2.6 per 100,000 inhabitants per year) hence it is difficult to compare frequency between different age groups. ${ }^{1}$ However, the few cases reported in literature and in our stroke center for patients over 70 years suggest that the frequency of diagnosis decreases with increasing age. CAD as a cause of stroke would appear to be rare in the elderly than in the younger patients as a peak of incidence is usually reported during the fourth and the fifth decades. ${ }^{1}$ In a recent study including 2,400 patients with proven CAD, only $7.4 \%$ of the patients were older than 60 years, confirming data from previous studies. ${ }^{12,13} \mathrm{CAD}$ is reported as a cause of cerebral infarction in $17 \%$ of young patients. ${ }^{14}$ The first theory explaining the higher incidence of CAD as an etiology of stroke in young populations is the modification of the vessel wall which becomes increasingly stiff with age. However, in a small series of consecutive patients with cerebral infarction due to $\mathrm{CAD}$, one-third were older than 60 years, suggesting that this cause of stroke is underestimated in the elderly in clinical practice. ${ }^{15}$ It is likely that CAD is more often diagnosed in younger patients who benefit from a more extensive diagnostic workup than the elderly. On the contrary, the increasing incidence of cerebral infarction with age due to common etiologies such as atherosclerosis and atrial fibrillation means that $\mathrm{CAD}$ is not often assessed in this population, and it is therefore less likely to be diagnosed.

It should be noted that neither of our patients presented with extensive atherosclerosis lesions. This may suggest that elderly patients with stroke due to CAD present fewer vascular risk factors and more recent infections (suspected of weakening the vessel wall) than those with other causes. ${ }^{16}$ However, for the time being, the absence of atherosclerosis as a predisposing factor of CAD for elderly patients can only be assumed. In patients older than 60 years with CAD, Ahl et al found at least two vascular risk factors in about $40 \%$, and Traenka et al found a higher rate of hypertension and hypercholesterolemia in comparison with younger patients. ${ }^{12,15}$ Vascular risk factors were also found in both of our patients. Both of them also presented former cerebral infarctions on FLAIR imaging showing that CAD can occur in a patient with previous vasculopathy. The second patient presented a chronic inflammatory disease which is thought to affect the vascular wall. The resulting CAD could be due to a transitory weakness of the arterial wall promoted by inflammation. ${ }^{17}$

In view of the cases we describe in this paper, some clinical signs caused by local compression of nerves surrounding vessels could suggest a diagnosis of CAD to physicians. First, headache is reported in most patients with $\mathrm{CAD}$, whatever their age, and more consistently than cervical pain. Even if headache is less present in patients older than 60 years than in younger patients, it remains a cornerstone of the diagnostic workup when CAD is suspected..$^{1,12}$ This is particularly true for elderly patients as it could also suggest other threatening causes like giant cell arteritis. This latter condition was ruled out for our first patient due to the absence of a biological inflammatory syndrome. The association of headache with Horner syndrome was typical of ICA dissection in the second case. Horner syndrome might be more frequent in older patients with CAD possibly due to weaker 
structure of the tissue surrounding the arteries and external compression of sympathetic nerve branches. Furthermore, CAD is more likely to involve the ICA in the elderly, and Horner syndrome is a clear diagnostic pointer. ${ }^{16}$ While the predominance of Horner syndrome in the elderly is not confirmed by recent studies, the sign is still found in $30 \%$ of the patients with CAD. ${ }^{12}$

In our first patient, CAD was suspected and confirmed through radiological arguments. Ultrasonography investigation is a non-invasive first-line examination. Some direct signs of vessel wall damage due to the tear (double-lumen sign and intimal flap) and the ensuing hematoma (intramural hematoma, dilation, tapered stenosis, and occlusion) can be observed. ${ }^{18}$ However, some ultrasonographic findings can be highly inconstant especially for CAD located at the distal part of the cervical artery and subadventitial hematoma. Moreover, it may be difficult to differentiate hematoma due to $\mathrm{CAD}$ from ruptured atherosclerotic plaque. As observed in our first patient, this investigation sometimes reveals only indirect signs with changes in blood flow patterns and increased resistive index (Figure 1). ${ }^{19}$ Once again, these findings are present only for CAD with hemodynamic consequences and a normal ultrasonography investigation does not exclude CAD. Diagnosis is confirmed through CT or MR angiography which are able to demonstrate typical aspects such as long tight stenosis with location remote from the carotid bulb (case 1, Figure 2A), and dilation with increased vessel diameter (also known as false aneurysm). Association of both signs is highly suggestive of the diagnosis of CAD and is usually called the pearl-and-string sign (case 2, Figure 4). ${ }^{20}$ Direct visualization of the intimal flap and false lumen is characteristic but more uncommon. The most relevant sign remains the identification of intramural hematoma for which axial fat-suppressed T1-weighted MRI is the most sensitive examination (case 1, Figure 2C). ${ }^{1,20}$ Changes in radiological signs can also be suggestive of CAD. Hematoma of the arterial wall can lead to rapid occlusion or, on the contrary, to recanalization, which is not common for arteriosclerotic lesions. ${ }^{21}$ The radiological signs described in our cases would appear to be similar to those usually encountered in younger patients.

From our study, we have very few data about treatments and outcome. Endovascular treatment is reported only once for a patient with a giant pseudoaneurysm with local compression. $^{2}$ Two patients received transitory anticoagulant therapy to lower the risk of embolic cerebral infarction, although the effectiveness of this approach remains to be demonstrated by studies. Indeed, the rate of events including cerebral ischemic events, hemorrhage in general, and death in studies comparing outcome of CAD patients treated with anticoagulant versus antiplatelet therapy is low (not $>3 \%){ }^{22}$ Consequently, it is difficult to demonstrate a significant difference between these two preventive strategies. Outcome seems to depend on the initial cerebral damage caused by CAD. None of the patients from our study experienced cerebral ischemic recurrence despite left ICA occlusion in one. However, it should be kept in mind that the overall recurrence rate of $\mathrm{CAD}$ reported in literature is as high as $25 \%{ }^{1}$

\section{Conclusions}

CAD appears all the rarer with increased age. Nevertheless, it should not be overlooked in old patients with vascular risk factors. It is almost certainly underdiagnosed in this population due to a higher prevalence of other common causes of stroke within this age range. However, CAD in the elderly could be more accurately detected provided that physicians keep this diagnosis in mind during the neurological examination. Clinical signs like headache at stroke onset or Horner syndrome should be assessed and, in the absence of another obvious cause, lead to MR or CT angiography of the cervical arteries. Radiological features of CAD in the elderly do not differ from those found in younger patients. Prospective observational studies are required in this specific population to assess therapy and outcome.

\section{Acknowledgment}

We acknowledge Felicity Neilson, of Matrix Consultants, for having reviewed the English language with scientific expertise.

\section{Disclosure}

The authors report no conflicts of interest in this work.

\section{References}

1. Debette S, Leys D. Cervical-artery dissections: predisposing factors, diagnosis, and outcome. Lancet Neurol. 2009;8(7):668-678.

2. Berkmen T, Troffkin N, Wakhloo AK. Direct percutaneous puncture of a cervical internal carotid artery aneurysm for coil placement after previous incomplete stent-assisted endovascular treatment. AJNR Am J Neuroradiol. 2003;24(6):1230-1233.

3. Mandrioli J, Zini A, Cavalleri F, Nichelli P, Panzetti P. Bilateral posterior medullary and cervical stroke: a case report. Neurol Sci. 2006; 27(4):281-283.

4. Albuquerque FC, Hu YC, Dashti SR, et al. Craniocervical arterial dissections as sequelae of chiropractic manipulation: patterns of injury and management. J Neurosurg. 2011;115(6):1197-1205.

5. Ast G, Woimant F, Georges B, Laurian C, Haguenau M. Spontaneous dissection of the internal carotid artery in 68 patients. Eur J Med. 1993; 2(8):466-472.

6. Buss A, Pech K, Roelver S, Bloemeke B, Klotzsch C, Breuer S. Functional polymorphisms in matrix metalloproteinases-1, $-3,-9$ and -12 in relation to cervical artery dissection. BMC Neurol. 2009;9:40. 
7. Huang YC, Chen YF, Wang YH, Tu YK, Jeng JS, Liu HM. Cervicocranial arterial dissection: experience of 73 patients in a single center. Surg Neurol. 2009;72(Supp1 2):S20-S27.

8. Kremer C, Mosso M, Georgiadis D, et al. Carotid dissection with permanent and transient occlusion or severe stenosis: long-term outcome. Neurology. 2003;60(2):271-275.

9. Schievink WI, Mokri B, O'Fallon WM. Recurrent spontaneous cervicalartery dissection. N Engl J Med. 1994;330(6):393-397.

10. Shibata T, Kubo M, Kuwayama N, Hirashima Y, Endo S. Warning headache of subarachnoid hemorrhage and infarction due to vertebrobasilar artery dissection. Clin J Pain. 2006;22(2):193-196.

11. Zach V, Zhovtis S, Kirchoff-Torres KF, Weinberger JM. Common carotid artery dissection: a case report and review of the literature. J Stroke Cerebrovasc Dis. 2012;21(1):52-60.

12. Traenka C, Dougoud D, Simonetti BG, et al; CADISP-Plus Study Group. Cervical artery dissection in patients $\geq 60$ years: often painless, few mechanical triggers. Neurology. 2017;88(14):1313-1320.

13. Debette S, Metso TM, Pezzini A, et al; CADISP-group. CADISPgenetics: an International project searching for genetic risk factors of cervical artery dissections. Int J Stroke. 2009;4(3):224-230.

14. Goeggel Simonetti B, Mono ML, Huynh-Do U, et al. Risk factors, aetiology and outcome of ischaemic stroke in young adults: the Swiss Young Stroke Study (SYSS). J Neurol. 2015;262(9):2025-2032.

15. Ahl B, Bokemeyer M, Ennen JC, Kohlmetz C, Becker H, Weissenborn K. Dissection of the brain supplying arteries over the life span. J Neurol Neurosurg Psychiatr. 2004;75(8):1194-1196.
16. Metso TM, Debette S, Grond-Ginsbach C, et al. Age-dependent differences in cervical artery dissection. J Neurol. 2012;259(10): 2202-2210.

17. Pfefferkorn T, Saam T, Rominger A, et al. Vessel wall inflammation in spontaneous cervical artery dissection: a prospective, observational positron emission tomography, computed tomography, and magnetic resonance imaging study. Stroke. 2011;42(6):1563-1568.

18. Suzuki R, Koga M, Toyoda K, et al. Identification of internal carotid artery dissection by transoral carotid ultrasonography. Cerebrovasc Dis. 2012;33(4):369-377.

19. Alecu C, Fortrat JO, Ducrocq X, Vespignani H, de Bray JM. Duplex scanning diagnosis of internal carotid artery dissections. A case control study. Cerebrovasc Dis. 2007;23(5-6):441-447.

20. Ben Hassen W, Machet A, Edjlali-Goujon M, et al. Imaging of cervical artery dissection. Diagn Interv Imaging. 2014;95(12):1151-1161.

21. Lacour JC, Ducrocq X, Vespignani H. Dissections of the carotid and vertebrobasilar arteries. Rev Prat. 2004;54(10):1093-1100.

22. Markus HS, Hayter E, Levi C, Feldman A, Venables G, Norris J with CADISS trial investigators. Antiplatelet treatment compared with anticoagulation treatment for cervical artery dissection (CADISS): a randomised trial. Lancet Neurol. 2015;14(4):361-367.
Clinical Interventions in Aging

\section{Publish your work in this journal}

Clinical Interventions in Aging is an international, peer-reviewed journal focusing on evidence-based reports on the value or lack thereof of treatments intended to prevent or delay the onset of maladaptive correlates of aging in human beings. This journal is indexed on PubMed Central, MedLine,

\section{Dovepress}

CAS, Scopus and the Elsevier Bibliographic databases. The manuscript management system is completely online and includes a very quick and fair peer-review system, which is all easy to use. Visit http://www.dovepress. com/testimonials.php to read real quotes from published authors. 